Supporting Information for

\title{
High Ionization Potential Conjugated Polymers
}

\author{
Youngmi Kim, James E. Whitten,"** Timothy M. Swager*
}

Department of Chemistry and Institute for Soldier Nanotechnologies, Massachusetts Institute of Technology, 77 Massachusetts Avenue, Cambridge, Massachusetts 02139, 'Department of Chemistry and Center for Advanced Materials, University of Massachusetts Lowell, One University Avenue, Lowell, MA 01854

X-ray crystal structure determination. Crystals suitable for X-ray analysis were grown by slow evaporation of $\mathrm{CH}_{2} \mathrm{Cl}_{2}-\mathrm{Hexane}$ solutions of one isomer of 3-a and 3-b, respectively. Crystal structure determination was performed using a Bruker Smart diffractometer equipped with a Kappa CCD area detector using radiation from a Mo- $\mathrm{K}_{\alpha}$ source monochromated through graphite $(\lambda=0.71073 \AA)$. Structures were solved and refined using the Bruker SHELXTL package. Crystal structure data are reproduced below (Table $1-9$ ). 
Table 1. Crystal data and structure refinement for Compound 3-a and 3-b.

\begin{tabular}{|c|c|c|}
\hline & 3-a & 3-b \\
\hline Empirical formula & $\mathrm{C} 39 \mathrm{H} 28 \mathrm{Cl} 2 \mathrm{O} 8$ & $\mathrm{C}_{34,20} \mathrm{H}_{14,40} \mathrm{Cl}_{0,40} \mathrm{~F}_{12}$ \\
\hline Formula weight & 695.51 & 667.44 \\
\hline Temperature & $100(2) \mathrm{K}$ & 193(2) K \\
\hline Wavelength & $0.71073 \AA$ & $0.71073 \AA$ \\
\hline Crystal system & Monoclinic & Monoclinic \\
\hline Space group & $\mathrm{C} 2 / \mathrm{c}$ & $\mathrm{P} 2(1) / \mathrm{n}$ \\
\hline Unit cell dimensions & $\begin{array}{l}\mathrm{a}=23.75(2) \AA \alpha \quad \alpha=90^{\circ} . \\
\mathrm{b}=8.456(9) \AA \beta=118.39^{\circ} . \\
\mathrm{c}=18.780(20) \AA \quad \AA=90^{\circ} .\end{array}$ & $\begin{array}{l}\mathrm{a}=8.4828(8) \AA \alpha=90^{\circ} \\
\mathrm{b}=13.0644(13) \AA \beta=101.579 \\
\mathrm{c}=13.703(2) \AA \quad \AA=90^{\circ}\end{array}$ \\
\hline Volume & $3318(6) \AA^{3}$ & $1487.7(3) \AA^{3}$ \\
\hline $\mathrm{Z}$ & 4 & 2 \\
\hline Density (calculated) & $1.392 \mathrm{Mg} / \mathrm{m}^{3}$ & $1.490 \mathrm{Mg} / \mathrm{m}^{3}$ \\
\hline Absorption coefficient & $0.251 \mathrm{~mm}^{-1}$ & $0.174 \mathrm{~mm}^{-1}$ \\
\hline $\mathrm{F}(000)$ & 1440 & 669 \\
\hline Crystal size & $0.20 \times 0.15 \times 0.10 \mathrm{~mm}^{3}$ & $0.22 \times 0.21 \times 0.16 \mathrm{~mm}^{3}$ \\
\hline $\begin{array}{l}\text { Theta range for data } \\
\text { collection }\end{array}$ & 1.95 to $24.99^{\circ}$. & 2.18 to $28.28^{\circ}$ \\
\hline Index ranges & $\begin{array}{l}-28<=\mathrm{h}<=24,0<=\mathrm{k}<=10, \\
0<=1<=22\end{array}$ & $\begin{array}{l}-11<=\mathrm{h}<=11,-15<=\mathrm{k}<=17, \\
-17<=1<=8\end{array}$ \\
\hline Reflections collected & 2907 & 7783 \\
\hline Independent reflections & $2907[\mathrm{R}$ (int) $=0.0509]$ & $3466[\mathrm{R}(\mathrm{int})=0.0170]$ \\
\hline $\begin{array}{l}\text { Completeness to theta }= \\
24.99^{\circ}\end{array}$ & $99.9 \%$ & $93.8 \%$ \\
\hline Absorption correction & $\begin{array}{l}\text { Semi-empirical from } \\
\text { equivalents }\end{array}$ & None \\
\hline $\begin{array}{l}\text { Max. and min. } \\
\text { transmission }\end{array}$ & 0.9753 and 0.9515 & \\
\hline Refinement method & $\begin{array}{l}\text { Full-matrix least-squares on } \\
\mathrm{F}^{2}\end{array}$ & $\begin{array}{l}\text { Full-matrix least-squares on } \\
\mathrm{F}^{2}\end{array}$ \\
\hline $\begin{array}{l}\text { Data / restraints / } \\
\text { parameters }\end{array}$ & 2907 / 0 / 224 & 3466 / 0 / 281 \\
\hline Goodness-of-fit on $\mathrm{F}^{2}$ & 1.211 & 1.174 \\
\hline $\begin{array}{l}\text { Final R indices } \\
{[\mathrm{I}>2 \operatorname{sigma}(\mathrm{I})]}\end{array}$ & $\mathrm{R} 1=0.0544, \mathrm{wR} 2=0.1243$ & $\mathrm{R} 1=0.0539, \mathrm{wR} 2=0.1445$ \\
\hline $\mathrm{R}$ indices (all data) & $\mathrm{R} 1=0.0566, \mathrm{wR} 2=0.1258$ & $\mathrm{R} 1=0.0612, w \mathrm{R} 2=0.1491$ \\
\hline $\begin{array}{l}\text { Largest diff. peak and } \\
\text { hole }\end{array}$ & 0.415 and -0.263 e. $\AA^{-3}$ & 0.510 and -0.324 e. $\AA^{-3}$ \\
\hline
\end{tabular}


Table 2. Selected bond lengths $[\AA]$ and angles $\left[{ }^{\circ}\right]$ for compound 3-a.

\begin{tabular}{|c|c|c|c|}
\hline \multicolumn{2}{|c|}{ Selected bond lengths $[\AA]$} & \multicolumn{2}{|c|}{ Selected bond angles $\left[{ }^{\circ}\right]$} \\
\hline $\mathrm{O}(1)-\mathrm{C}(16)$ & $1.207(3)$ & $\mathrm{C}(16)-\mathrm{O}(2)-\mathrm{C}(17)$ & $115.9(2)$ \\
\hline $\mathrm{O}(2)-\mathrm{C}(16)$ & $1.331(3)$ & $\mathrm{C}(18)-\mathrm{O}(4)-\mathrm{C}(19)$ & $115.5(2)$ \\
\hline $\mathrm{O}(2)-\mathrm{C}(17)$ & $1.442(3)$ & $C(2)-C(1)-C(16)$ & $126.4(2)$ \\
\hline $\mathrm{O}(3)-\mathrm{C}(18)$ & $1.197(3)$ & $C(2)-C(1)-C(6)$ & $113.9(2)$ \\
\hline $\mathrm{O}(4)-\mathrm{C}(18)$ & $1.328(3)$ & $C(16)-C(1)-C(6)$ & $119.6(2)$ \\
\hline $\mathrm{O}(4)-\mathrm{C}(19)$ & $1.446(3)$ & $C(1)-C(2)-C(18)$ & $128.3(2)$ \\
\hline$C(1)-C(2)$ & $1.332(4)$ & $\mathrm{C}(1)-\mathrm{C}(2)-\mathrm{C}(3)$ & $113.8(2)$ \\
\hline$C(1)-C(16)$ & $1.474(3)$ & $\mathrm{C}(18)-\mathrm{C}(2)-\mathrm{C}(3)$ & $117.9(2)$ \\
\hline$C(1)-C(6)$ & $1.527(3)$ & $\mathrm{C}(4)-\mathrm{C}(3)-\mathrm{C}(7)$ & $105.82(19)$ \\
\hline $\mathrm{C}(2)-\mathrm{C}(18)$ & $1.488(4)$ & $\mathrm{C}(4)-\mathrm{C}(3)-\mathrm{C}(2)$ & $105.16(19)$ \\
\hline$C(2)-C(3)$ & $1.527(3)$ & $\mathrm{C}(7)-\mathrm{C}(3)-\mathrm{C}(2)$ & $105.62(19)$ \\
\hline $\mathrm{C}(3)-\mathrm{C}(4)$ & $1.521(3)$ & $C(5)-C(4)-C(13) \# 1$ & $121.3(2)$ \\
\hline$C(3)-C(7)$ & $1.526(4)$ & $C(5)-C(4)-C(3)$ & $113.0(2)$ \\
\hline$C(4)-C(5)$ & $1.386(3)$ & $\mathrm{C}(13) \# 1-\mathrm{C}(4)-\mathrm{C}(3)$ & $125.6(2)$ \\
\hline $\mathrm{C}(4)-\mathrm{C}(13) \# 1$ & $1.392(3)$ & $C(4)-C(5)-C(13)$ & $121.4(2)$ \\
\hline$C(5)-C(13)$ & $1.399(3)$ & $C(4)-C(5)-C(6)$ & $112.7(2)$ \\
\hline$C(5)-C(6)$ & $1.520(3)$ & $C(13)-C(5)-C(6)$ & $125.9(2)$ \\
\hline$C(6)-C(8)$ & $1.526(3)$ & $C(5)-C(6)-C(8)$ & $105.11(19)$ \\
\hline$C(7)-C(12)$ & $1.384(4)$ & $C(5)-C(6)-C(1)$ & $105.55(19)$ \\
\hline $\mathrm{C}(7)-\mathrm{C}(8)$ & $1.391(4)$ & $\mathrm{C}(8)-\mathrm{C}(6)-\mathrm{C}(1)$ & $106.0(2)$ \\
\hline $\mathrm{C}(8)-\mathrm{C}(9)$ & $1.380(4)$ & $C(12)-C(7)-C(8)$ & $121.0(2)$ \\
\hline $\mathrm{C}(9)-\mathrm{C}(10)$ & $1.387(4)$ & $C(12)-C(7)-C(3)$ & $126.4(2)$ \\
\hline $\mathrm{C}(10)-\mathrm{C}(11)$ & $1.385(4)$ & $\mathrm{C}(8)-\mathrm{C}(7)-\mathrm{C}(3)$ & $112.6(2)$ \\
\hline$C(11)-C(12)$ & $1.392(4)$ & $\mathrm{C}(9)-\mathrm{C}(8)-\mathrm{C}(7)$ & $120.2(2)$ \\
\hline$C(13)-C(4) \# 1$ & $1.392(3)$ & $\mathrm{C}(9)-\mathrm{C}(8)-\mathrm{C}(6)$ & $127.0(2)$ \\
\hline$C(13)-C(14)$ & $1.439(4)$ & $C(7)-C(8)-C(6)$ & $112.8(2)$ \\
\hline \multirow[t]{6}{*}{$C(14)-C(15)$} & $1.180(4)$ & $\mathrm{C}(8)-\mathrm{C}(9)-\mathrm{C}(10)$ & $119.4(2)$ \\
\hline & & $\mathrm{C}(11)-\mathrm{C}(10)-\mathrm{C}(9)$ & $120.2(2)$ \\
\hline & & $\mathrm{C}(10)-\mathrm{C}(11)-\mathrm{C}(12)$ & $120.9(2)$ \\
\hline & & $\mathrm{C}(7)-\mathrm{C}(12)-\mathrm{C}(11)$ & $118.4(2)$ \\
\hline & & $\mathrm{C}(4) \# 1-\mathrm{C}(13)-\mathrm{C}(5)$ & $117.3(2)$ \\
\hline & & $\mathrm{C}(4) \# 1-\mathrm{C}(13)-\mathrm{C}(14)$ & $121.2(2)$ \\
\hline
\end{tabular}




\begin{tabular}{ll}
\hline $\mathrm{C}(5)-\mathrm{C}(13)-\mathrm{C}(14)$ & $121.5(2)$ \\
$\mathrm{C}(15)-\mathrm{C}(14)-\mathrm{C}(13)$ & $177.9(3)$ \\
$\mathrm{O}(1)-\mathrm{C}(16)-\mathrm{O}(2)$ & $124.4(2)$ \\
$\mathrm{O}(1)-\mathrm{C}(16)-\mathrm{C}(1)$ & $123.4(2)$ \\
$\mathrm{O}(2)-\mathrm{C}(16)-\mathrm{C}(1)$ & $112.2(2)$ \\
$\mathrm{O}(3)-\mathrm{C}(18)-\mathrm{O}(4)$ & $124.9(2)$ \\
$\mathrm{O}(3)-\mathrm{C}(18)-\mathrm{C}(2)$ & $123.7(2)$ \\
$\mathrm{O}(4)-\mathrm{C}(18)-\mathrm{C}(2)$ & $111.3(2)$ \\
\hline
\end{tabular}

Symmetry transformations used to generate equivalent atoms:

\#1 -x-1/2,-y+1/2,-z 
Table 3. Selected bond lengths $[\AA ̊]$ and angles $\left[^{\circ}\right]$ for compound 3-b.

\begin{tabular}{|c|c|c|c|}
\hline \multicolumn{2}{|c|}{ Selected bond lengths $[\AA]$} & \multicolumn{2}{|c|}{ Selected bond angles $\left[^{\circ}\right]$} \\
\hline $\mathrm{C}(1)-\mathrm{C}(2)$ & $1.186(2)$ & $\mathrm{C}(1)-\mathrm{C}(2)-\mathrm{C}(3)$ & $175.93(17)$ \\
\hline $\mathrm{C}(2)-\mathrm{C}(3)$ & $1.438(2)$ & $\mathrm{C}(4)-\mathrm{C}(3)-\mathrm{C}(5)$ & $116.86(13)$ \\
\hline $\mathrm{C}(3)-\mathrm{C}(4)$ & $1.402(2)$ & $\mathrm{C}(4)-\mathrm{C}(3)-\mathrm{C}(2)$ & $120.29(13)$ \\
\hline $\mathrm{C}(3)-\mathrm{C}(5)$ & $1.406(2)$ & $\mathrm{C}(5)-\mathrm{C}(3)-\mathrm{C}(2)$ & $122.81(13)$ \\
\hline $\mathrm{C}(4)-\mathrm{C}(5) \# 1$ & $1.391(2)$ & $\mathrm{C}(5) \# 1-\mathrm{C}(4)-\mathrm{C}(3)$ & $122.03(13)$ \\
\hline $\mathrm{C}(4)-\mathrm{C}(9) \# 1$ & $1.527(2)$ & $\mathrm{C}(5) \# 1-\mathrm{C}(4)-\mathrm{C}(9) \# 1$ & $112.76(13)$ \\
\hline $\mathrm{C}(5)-\mathrm{C}(4) \# 1$ & $1.392(2)$ & $\mathrm{C}(3)-\mathrm{C}(4)-\mathrm{C}(9) \# 1$ & $125.21(13)$ \\
\hline$C(5)-C(6)$ & $1.529(2)$ & $\mathrm{C}(4) \# 1-\mathrm{C}(5)-\mathrm{C}(3)$ & $121.10(13)$ \\
\hline$C(6)-C(15)$ & $1.527(2)$ & $C(4) \# 1-C(5)-C(6)$ & $112.66(13)$ \\
\hline$C(6)-C(7)$ & $1.537(2)$ & $C(3)-C(5)-C(6)$ & $126.23(13)$ \\
\hline $\mathrm{C}(7)-\mathrm{C}(8)$ & $1.330(2)$ & $C(15)-C(6)-C(5)$ & $106.22(12)$ \\
\hline$C(7)-C(16)$ & $1.501(2)$ & $C(15)-C(6)-C(7)$ & $105.46(12)$ \\
\hline $\mathrm{C}(8)-\mathrm{C}(17)$ & $1.503(2)$ & $C(5)-C(6)-C(7)$ & $105.24(12)$ \\
\hline $\mathrm{C}(8)-\mathrm{C}(9)$ & $1.533(2)$ & $C(8)-C(7)-C(16)$ & $129.04(16)$ \\
\hline $\mathrm{C}(9)-\mathrm{C}(10)$ & $1.526(2)$ & $\mathrm{C}(8)-\mathrm{C}(7)-\mathrm{C}(6)$ & $113.82(14)$ \\
\hline $\mathrm{C}(9)-\mathrm{C}(4) \# 1$ & $1.527(2)$ & $C(16)-C(7)-C(6)$ & $117.13(14)$ \\
\hline $\mathrm{C}(10)-\mathrm{C}(11)$ & $1.381(2)$ & $\mathrm{C}(7)-\mathrm{C}(8)-\mathrm{C}(17)$ & $127.32(17)$ \\
\hline $\mathrm{C}(10)-\mathrm{C}(15)$ & $1.396(2)$ & $\mathrm{C}(7)-\mathrm{C}(8)-\mathrm{C}(9)$ & $113.84(14)$ \\
\hline$C(11)-C(12)$ & $1.396(3)$ & $\mathrm{C}(17)-\mathrm{C}(8)-\mathrm{C}(9)$ & $118.82(16)$ \\
\hline $\mathrm{C}(12)-\mathrm{C}(13)$ & $1.381(3)$ & $\mathrm{C}(10)-\mathrm{C}(9)-\mathrm{C}(4) \# 1$ & $105.55(12)$ \\
\hline$C(13)-C(14)$ & $1.394(3)$ & $\mathrm{C}(10)-\mathrm{C}(9)-\mathrm{C}(8)$ & $106.34(12)$ \\
\hline$C(14)-C(15)$ & $1.385(2)$ & C(4)\#1-C(9)-C(8) & $105.07(12)$ \\
\hline $\mathrm{C}(16)-\mathrm{F}\left(1^{\prime}\right)$ & $1.154(7)$ & $C(11)-C(10)-C(15)$ & $120.82(15)$ \\
\hline $\mathrm{C}(16)-\mathrm{F}\left(3^{\prime}\right)$ & $1.258(9)$ & $C(11)-C(10)-C(9)$ & $126.39(15)$ \\
\hline $\mathrm{C}(16)-\mathrm{F}(1)$ & $1.286(3)$ & $\mathrm{C}(15)-\mathrm{C}(10)-\mathrm{C}(9)$ & $112.78(13)$ \\
\hline$C(16)-F(2)$ & $1.317(3)$ & $\mathrm{C}(10)-\mathrm{C}(11)-\mathrm{C}(12)$ & $118.86(17)$ \\
\hline $\mathrm{C}(16)-\mathrm{F}(3)$ & $1.357(3)$ & $\mathrm{C}(13)-\mathrm{C}(12)-\mathrm{C}(11)$ & $120.33(17)$ \\
\hline $\mathrm{C}(16)-\mathrm{F}\left(2^{\prime}\right)$ & $1.454(9)$ & $C(12)-C(13)-C(14)$ & $120.95(17)$ \\
\hline $\mathrm{C}(17)-\mathrm{F}\left(6^{\prime}\right)$ & $1.149(12)$ & $C(15)-C(14)-C(13)$ & $118.73(17)$ \\
\hline $\mathrm{C}(17)-\mathrm{F}\left(5^{\prime}\right)$ & $1.156(12)$ & $\mathrm{C}(14)-\mathrm{C}(15)-\mathrm{C}(10)$ & $120.29(15)$ \\
\hline $\mathrm{C}(17)-\mathrm{F}(4)$ & $1.295(4)$ & $\mathrm{C}(14)-\mathrm{C}(15)-\mathrm{C}(6)$ & $127.19(15)$ \\
\hline$C(17)-F(5)$ & $1.300(3)$ & $C(10)-C(15)-C(6)$ & $112.52(13)$ \\
\hline $\mathrm{C}(17)-\mathrm{F}(6)$ & $1.372(3)$ & $\mathrm{F}\left(1^{\prime}\right)-\mathrm{C}(16)-\mathrm{F}\left(3^{\prime}\right)$ & $117.3(10)$ \\
\hline $\mathrm{C}(17)-\mathrm{F}\left(4^{\prime}\right)$ & $1.511(9)$ & $\mathrm{F}\left(1^{\prime}\right)-\mathrm{C}(16)-\mathrm{F}(1)$ & $125.7(4)$ \\
\hline $\mathrm{F}(1)-\mathrm{F}\left(3^{\prime}\right)$ & $0.809(12)$ & $\mathrm{F}\left(3^{\prime}\right)-\mathrm{C}(16)-\mathrm{F}(1)$ & $37.1(6)$ \\
\hline $\mathrm{F}(1)-\mathrm{F}\left(2^{\prime}\right)$ & $1.428(9)$ & $\mathrm{F}\left(1^{\prime}\right)-\mathrm{C}(16)-\mathrm{F}(2)$ & $44.8(10)$ \\
\hline $\mathrm{F}(2)-\mathrm{F}\left(1^{\prime}\right)$ & $0.953(19)$ & $\mathrm{F}\left(3^{\prime}\right)-\mathrm{C}(16)-\mathrm{F}(2)$ & $134.0(4)$ \\
\hline $\mathrm{F}(2)-\mathrm{F}\left(2^{\prime}\right)$ & $1.344(12)$ & $\mathrm{F}(1)-\mathrm{C}(16)-\mathrm{F}(2)$ & $110.4(3)$ \\
\hline $\mathrm{F}(3)-\mathrm{F}\left(1^{\prime}\right)$ & $1.214(19)$ & $\mathrm{F}\left(1^{\prime}\right)-\mathrm{C}(16)-\mathrm{F}(3)$ & $57.1(10)$ \\
\hline $\mathrm{F}(3)-\mathrm{F}\left(3^{\prime}\right)$ & $1.561(15)$ & $\mathrm{F}\left(3^{\prime}\right)-\mathrm{C}(16)-\mathrm{F}(3)$ & $73.2(7)$ \\
\hline $\mathrm{F}(4)-\mathrm{F}\left(6^{\prime}\right)$ & $0.57(3)$ & $\mathrm{F}(1)-\mathrm{C}(16)-\mathrm{F}(3)$ & $106.0(3)$ \\
\hline $\mathrm{F}(4)-\mathrm{F}\left(4^{\prime}\right)$ & $1.508(11)$ & $\mathrm{F}(2)-\mathrm{C}(16)-\mathrm{F}(3)$ & $101.4(2)$ \\
\hline
\end{tabular}




\begin{tabular}{|c|c|c|c|}
\hline $\mathrm{F}(5)-\mathrm{F}\left(5^{\prime}\right)$ & $0.93(3)$ & $\mathrm{F}\left(1^{\prime}\right)-\mathrm{C}(16)-\mathrm{F}\left(2^{\prime}\right)$ & 99.4(9) \\
\hline $\mathrm{F}(5)-\mathrm{F}\left(4^{\prime}\right)$ & $1.397(13)$ & $\mathrm{F}\left(3^{\prime}\right)-\mathrm{C}(16)-\mathrm{F}\left(2^{\prime}\right)$ & $99.1(7)$ \\
\hline $\mathrm{F}(6)-\mathrm{F}\left(5^{\prime}\right)$ & $1.31(3)$ & $\mathrm{F}(1)-\mathrm{C}(16)-\mathrm{F}\left(2^{\prime}\right)$ & $62.5(4)$ \\
\hline $\mathrm{F}(6)-\mathrm{F}\left(6^{\prime}\right)$ & $1.56(3)$ & $\mathrm{F}(2)-\mathrm{C}(16)-\mathrm{F}\left(2^{\prime}\right)$ & $57.7(5)$ \\
\hline $\mathrm{C}(18 \mathrm{~S})-\mathrm{Cl}$ & $1.68(2)$ & $\mathrm{F}(3)-\mathrm{C}(16)-\mathrm{F}\left(2^{\prime}\right)$ & $143.2(4)$ \\
\hline $\mathrm{C}(18 \mathrm{~S})-\mathrm{Cl} 2$ & $1.716(19)$ & $\mathrm{F}\left(1^{\prime}\right)-\mathrm{C}(16)-\mathrm{C}(7)$ & $120.6(4)$ \\
\hline \multirow[t]{40}{*}{$\mathrm{Cl}-\mathrm{C}(18 \mathrm{~S}) \# 2$} & $1.716(19)$ & $\mathrm{F}\left(3^{\prime}\right)-\mathrm{C}(16)-\mathrm{C}(7)$ & $110.1(5)$ \\
\hline & & $\mathrm{F}(1)-\mathrm{C}(16)-\mathrm{C}(7)$ & 113.73(19) \\
\hline & & $\mathrm{F}(2)-\mathrm{C}(16)-\mathrm{C}(7)$ & $114.4(2)$ \\
\hline & & $\mathrm{F}(3)-\mathrm{C}(16)-\mathrm{C}(7)$ & 109.91(16) \\
\hline & & $\mathrm{F}\left(2^{\prime}\right)-\mathrm{C}(16)-\mathrm{C}(7)$ & $106.5(4)$ \\
\hline & & $\mathrm{F}\left(6^{\prime}\right)-\mathrm{C}(17)-\mathrm{F}\left(5^{\prime}\right)$ & $117.4(14)$ \\
\hline & & $\mathrm{F}\left(6^{\prime}\right)-\mathrm{C}(17)-\mathrm{F}(4)$ & $26.0(14)$ \\
\hline & & $\mathrm{F}\left(5^{\prime}\right)-\mathrm{C}(17)-\mathrm{F}(4)$ & $129.6(8)$ \\
\hline & & $\mathrm{F}\left(6^{\prime}\right)-\mathrm{C}(17)-\mathrm{F}(5)$ & $122.2(9)$ \\
\hline & & $\mathrm{F}\left(5^{\prime}\right)-\mathrm{C}(17)-\mathrm{F}(5)$ & $43.8(16)$ \\
\hline & & $\mathrm{F}(4)-\mathrm{C}(17)-\mathrm{F}(5)$ & $112.3(3)$ \\
\hline & & $\mathrm{F}\left(6^{\prime}\right)-\mathrm{C}(17)-\mathrm{F}(6)$ & 76.1(14) \\
\hline & & $\mathrm{F}\left(5^{\prime}\right)-\mathrm{C}(17)-\mathrm{F}(6)$ & $61.9(17)$ \\
\hline & & $F(4)-C(17)-F(6)$ & $101.9(3)$ \\
\hline & & $\mathrm{F}(5)-\mathrm{C}(17)-\mathrm{F}(6)$ & $104.2(3)$ \\
\hline & & $\mathrm{F}\left(6^{\prime}\right)-\mathrm{C}(17)-\mathrm{C}(8)$ & 121.1(9) \\
\hline & & $\mathrm{F}\left(5^{\prime}\right)-\mathrm{C}(17)-\mathrm{C}(8)$ & $116.3(8)$ \\
\hline & & $\mathrm{F}(4)-\mathrm{C}(17)-\mathrm{C}(8)$ & $114.0(2)$ \\
\hline & & $\mathrm{F}(5)-\mathrm{C}(17)-\mathrm{C}(8)$ & $112.6(2)$ \\
\hline & & $\mathrm{F}(6)-\mathrm{C}(17)-\mathrm{C}(8)$ & $110.9(2)$ \\
\hline & & $\mathrm{F}\left(6^{\prime}\right)-\mathrm{C}(17)-\mathrm{F}\left(4^{\prime}\right)$ & $88.2(12)$ \\
\hline & & $\mathrm{F}\left(5^{\prime}\right)-\mathrm{C}(17)-\mathrm{F}\left(4^{\prime}\right)$ & $101.2(14)$ \\
\hline & & $\mathrm{F}(4)-\mathrm{C}(17)-\mathrm{F}\left(4^{\prime}\right)$ & $64.5(5)$ \\
\hline & & $\mathrm{F}(5)-\mathrm{C}(17)-\mathrm{F}\left(4^{\prime}\right)$ & $59.0(6)$ \\
\hline & & $\mathrm{F}(6)-\mathrm{C}(17)-\mathrm{F}\left(4^{\prime}\right)$ & $145.8(4)$ \\
\hline & & $\mathrm{C}(8)-\mathrm{C}(17)-\mathrm{F}\left(4^{\prime}\right)$ & $103.3(3)$ \\
\hline & & $\mathrm{F}\left(3^{\prime}\right)-\mathrm{F}(1)-\mathrm{C}(16)$ & $69.6(7)$ \\
\hline & & $\mathrm{F}\left(3^{\prime}\right)-\mathrm{F}(1)-\mathrm{F}\left(2^{\prime}\right)$ & $133.3(10)$ \\
\hline & & $\mathrm{C}(16)-\mathrm{F}(1)-\mathrm{F}\left(2^{\prime}\right)$ & $64.6(4)$ \\
\hline & & $\mathrm{F}\left(1^{\prime}\right)-\mathrm{F}(2)-\mathrm{C}(16)$ & $58.5(5)$ \\
\hline & & $\mathrm{F}\left(1^{\prime}\right)-\mathrm{F}(2)-\mathrm{F}\left(2^{\prime}\right)$ & $120.1(7)$ \\
\hline & & $\mathrm{C}(16)-\mathrm{F}(2)-\mathrm{F}\left(2^{\prime}\right)$ & $66.3(4)$ \\
\hline & & $\mathrm{F}\left(1^{\prime}\right)-\mathrm{F}(3)-\mathrm{C}(16)$ & $53.0(5)$ \\
\hline & & $\mathrm{F}\left(1^{\prime}\right)-\mathrm{F}(3)-\mathrm{F}\left(3^{\prime}\right)$ & $95.1(6)$ \\
\hline & & $\mathrm{C}(16)-\mathrm{F}(3)-\mathrm{F}\left(3^{\prime}\right)$ & $50.5(4)$ \\
\hline & & $\mathrm{F}\left(6^{\prime}\right)-\mathrm{F}(4)-\mathrm{C}(17)$ & $62.4(13)$ \\
\hline & & $\mathrm{F}\left(6^{\prime}\right)-\mathrm{F}(4)-\mathrm{F}\left(4^{\prime}\right)$ & $121.5(16)$ \\
\hline & & $\mathrm{C}(17)-\mathrm{F}(4)-\mathrm{F}\left(4^{\prime}\right)$ & $64.7(4)$ \\
\hline & & $\mathrm{F}\left(5^{\prime}\right)-\mathrm{F}(5)-\mathrm{C}(17)$ & $59.8(7)$ \\
\hline & & $\mathrm{F}\left(5^{\prime}\right)-\mathrm{F}(5)-\mathrm{F}\left(4^{\prime}\right)$ & $125.2(10)$ \\
\hline
\end{tabular}




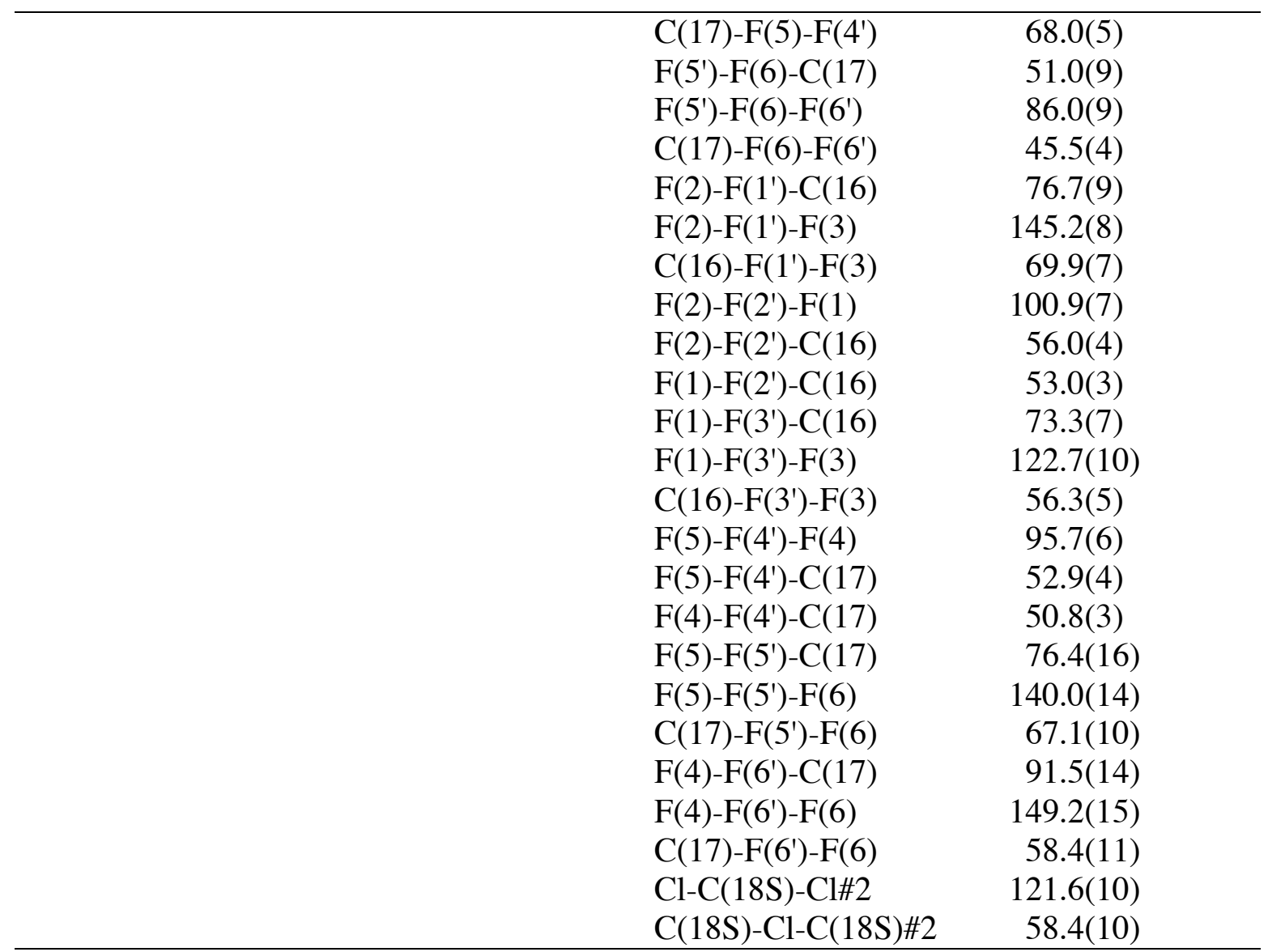

Symmetry transformations used to generate equivalent atoms:

$\# 1-\mathrm{x}+1,-\mathrm{y},-\mathrm{z}+1 \quad \# 2-\mathrm{x}+2,-\mathrm{y}+1,-\mathrm{z}+1$ 
Table 4. Atomic coordinates ( $\times 10^{4}$ ) and equivalent isotropic displacement parameters $\left(\AA^{2} \times 10^{3}\right)$ for compound 3-a. U(eq) is defined as one third of the trace of the orthogonalized $\mathrm{U}^{\mathrm{ij}}$ tensor.

\begin{tabular}{|c|c|c|c|c|}
\hline & $\mathrm{x}$ & $\mathrm{y}$ & $\mathrm{z}$ & $\mathrm{U}(\mathrm{eq})$ \\
\hline $\mathrm{O}(1)$ & $-9(1)$ & $1262(2)$ & 1152(1) & $20(1)$ \\
\hline $\mathrm{O}(2)$ & $-178(1)$ & $2820(2)$ & 101(1) & $20(1)$ \\
\hline $\mathrm{O}(3)$ & $-1452(1)$ & $3680(2)$ & $-1647(1)$ & $26(1)$ \\
\hline $\mathrm{O}(4)$ & $-894(1)$ & $1480(2)$ & $-1546(1)$ & $25(1)$ \\
\hline $\mathrm{C}(1)$ & $-1041(1)$ & 1374(3) & $19(1)$ & $14(1)$ \\
\hline $\mathrm{C}(2)$ & $-1417(1)$ & 1621(3) & $-769(1)$ & $15(1)$ \\
\hline $\mathrm{C}(3)$ & $-2102(1)$ & 1031(3) & $-1081(1)$ & $14(1)$ \\
\hline $\mathrm{C}(4)$ & $-2343(1)$ & 1854(3) & $-557(1)$ & $14(1)$ \\
\hline $\mathrm{C}(5)$ & $-1953(1)$ & 1602(3) & $264(1)$ & $13(1)$ \\
\hline $\mathrm{C}(6)$ & $-1378(1)$ & $567(3)$ & $440(1)$ & $14(1)$ \\
\hline$C(7)$ & $-2042(1)$ & $-715(3)$ & $-851(1)$ & $15(1)$ \\
\hline $\mathrm{C}(8)$ & $-1659(1)$ & $-962(3)$ & $-25(2)$ & $15(1)$ \\
\hline $\mathrm{C}(9)$ & $-1574(1)$ & $-2470(3)$ & 291(2) & $17(1)$ \\
\hline$C(10)$ & $-1859(1)$ & $-3738(3)$ & $-225(2)$ & $21(1)$ \\
\hline $\mathrm{C}(11)$ & $-2226(1)$ & $-3490(3)$ & $-1050(2)$ & $22(1)$ \\
\hline $\mathrm{C}(12)$ & $-2325(1)$ & $-1972(3)$ & $-1373(2)$ & $18(1)$ \\
\hline $\mathrm{C}(13)$ & $-2104(1)$ & $2237(3)$ & $840(1)$ & $13(1)$ \\
\hline $\mathrm{C}(14)$ & $-1712(1)$ & 1932(3) & 1691(2) & $15(1)$ \\
\hline$C(15)$ & $-1401(1)$ & 1641(3) & $2386(2)$ & $19(1)$ \\
\hline$C(16)$ & $-358(1)$ & 1792(3) & $489(1)$ & $15(1)$ \\
\hline $\mathrm{C}(17)$ & 489(1) & $3261(3)$ & $508(2)$ & $27(1)$ \\
\hline $\mathrm{C}(18)$ & $-1254(1)$ & 2407(3) & $-1357(1)$ & $16(1)$ \\
\hline$C(19)$ & $-717(2)$ & 2129(4) & $-2123(2)$ & $36(1)$ \\
\hline $\mathrm{C}(1 \mathrm{~S})$ & 0 & $3458(4)$ & 2500 & $26(1)$ \\
\hline $\mathrm{Cl}(1 \mathrm{~S})$ & $514(1)$ & $4623(1)$ & $3341(1)$ & $27(1)$ \\
\hline
\end{tabular}


Table 5. Anisotropic displacement parameters $\left(\AA^{2} \times 10^{3}\right)$ for compound 3-a. The anisotropic displacement factor exponent takes the form: $-2 \pi^{2}\left[h^{2} a^{* 2} U^{11}+\ldots+2 h k a^{*} b^{*}\right.$ $\left.\mathrm{U}^{12}\right]$.

\begin{tabular}{|c|c|c|c|c|c|c|}
\hline & $\mathrm{U}^{11}$ & $\mathrm{U}^{22}$ & $\mathrm{U}^{33}$ & $\mathrm{U}^{23}$ & $\mathrm{U}^{13}$ & $\mathrm{U}^{12}$ \\
\hline $\mathrm{O}(1)$ & $13(1)$ & $23(1)$ & $21(1)$ & $0(1)$ & $4(1)$ & $0(1)$ \\
\hline $\mathrm{O}(2)$ & $11(1)$ & $23(1)$ & $25(1)$ & $2(1)$ & $7(1)$ & $-5(1)$ \\
\hline $\mathrm{O}(3)$ & $27(1)$ & $22(1)$ & $30(1)$ & $8(1)$ & $14(1)$ & $5(1)$ \\
\hline $\mathrm{O}(4)$ & $32(1)$ & $21(1)$ & $33(1)$ & $0(1)$ & $25(1)$ & $0(1)$ \\
\hline $\mathrm{C}(1)$ & $12(1)$ & $11(1)$ & $22(1)$ & $-1(1)$ & $9(1)$ & $3(1)$ \\
\hline $\mathrm{C}(2)$ & $13(1)$ & $12(1)$ & $20(1)$ & $-3(1)$ & $8(1)$ & $1(1)$ \\
\hline$C(3)$ & 11(1) & $16(1)$ & $15(1)$ & $0(1)$ & $5(1)$ & $0(1)$ \\
\hline $\mathrm{C}(4)$ & $13(1)$ & $10(1)$ & $19(1)$ & $-2(1)$ & $8(1)$ & $-4(1)$ \\
\hline $\mathrm{C}(5)$ & $9(1)$ & $11(1)$ & $18(1)$ & $0(1)$ & $5(1)$ & $-2(1)$ \\
\hline$C(6)$ & 11(1) & $16(1)$ & $16(1)$ & $0(1)$ & $6(1)$ & $0(1)$ \\
\hline $\mathrm{C}(7)$ & $9(1)$ & $16(1)$ & $21(1)$ & $-1(1)$ & $9(1)$ & $1(1)$ \\
\hline $\mathrm{C}(8)$ & $8(1)$ & $17(1)$ & $22(1)$ & $-2(1)$ & $9(1)$ & $1(1)$ \\
\hline $\mathrm{C}(9)$ & $13(1)$ & $19(1)$ & $23(1)$ & $2(1)$ & $10(1)$ & $3(1)$ \\
\hline$C(10)$ & $17(1)$ & $15(1)$ & $35(2)$ & $3(1)$ & $16(1)$ & $1(1)$ \\
\hline $\mathrm{C}(11)$ & $20(1)$ & $17(1)$ & $33(2)$ & $-8(1)$ & $15(1)$ & $-5(1)$ \\
\hline$C(12)$ & $13(1)$ & $21(1)$ & $21(1)$ & $-2(1)$ & $8(1)$ & $-1(1)$ \\
\hline$C(13)$ & $10(1)$ & $12(1)$ & $16(1)$ & $0(1)$ & $5(1)$ & $-3(1)$ \\
\hline$C(14)$ & $10(1)$ & $12(1)$ & $24(1)$ & $-2(1)$ & $10(1)$ & $-1(1)$ \\
\hline$C(15)$ & $17(1)$ & $22(1)$ & $17(1)$ & $0(1)$ & $6(1)$ & $-1(1)$ \\
\hline$C(16)$ & $13(1)$ & $12(1)$ & $19(1)$ & $-4(1)$ & $8(1)$ & $2(1)$ \\
\hline$C(17)$ & $15(1)$ & $28(1)$ & $37(2)$ & $3(1)$ & $12(1)$ & $-5(1)$ \\
\hline$C(18)$ & $11(1)$ & $18(1)$ & $14(1)$ & $-4(1)$ & $2(1)$ & $-4(1)$ \\
\hline C(19) & $48(2)$ & $39(2)$ & $42(2)$ & $2(1)$ & $38(2)$ & $-2(1)$ \\
\hline $\mathrm{C}(1 \mathrm{~S})$ & $26(2)$ & $19(2)$ & $24(2)$ & 0 & $3(2)$ & 0 \\
\hline $\mathrm{Cl}(1 \mathrm{~S})$ & $26(1)$ & $24(1)$ & $26(1)$ & $-4(1)$ & $7(1)$ & $-6(1)$ \\
\hline
\end{tabular}


Table 6. Hydrogen coordinates ( x 104) and isotropic displacement parameters $\left(\AA^{2} \times 10^{3}\right)$ for compound 3-a.

\begin{tabular}{lrrrr}
\hline & $\mathrm{x}$ & $\mathrm{y}$ & $\mathrm{z}$ & $\mathrm{U}(\mathrm{eq})$ \\
\hline $\mathrm{H}(3)$ & -2380 & 1220 & -1673 & 17 \\
$\mathrm{H}(6)$ & -1091 & 394 & 1031 & 17 \\
$\mathrm{H}(9)$ & -1323 & -2637 & 857 & 21 \\
$\mathrm{H}(10)$ & -1801 & -4780 & -12 & 25 \\
$\mathrm{H}(11)$ & -2413 & -4368 & -1399 & 27 \\
$\mathrm{H}(12)$ & -2581 & -1802 & -1938 & 22 \\
$\mathrm{H}(15)$ & -1151 & 1406 & 2945 & 23 \\
$\mathrm{H}(17 \mathrm{~A})$ & 592 & 3769 & 1025 & 40 \\
$\mathrm{H}(17 \mathrm{~B})$ & 574 & 4000 & 168 & 40 \\
$\mathrm{H}(17 \mathrm{C})$ & 754 & 2314 & 608 & 40 \\
$\mathrm{H}(19 \mathrm{~A})$ & -1104 & 2406 & -2623 & 55 \\
$\mathrm{H}(19 B)$ & -470 & 1343 & -2243 & 55 \\
$\mathrm{H}(19 \mathrm{C})$ & -455 & 3079 & -1896 & 55 \\
$\mathrm{H}(1 \mathrm{~S} 1)$ & -261 & 2769 & 2655 & 32 \\
$\mathrm{H}(1 \mathrm{~S} 2)$ & 261 & 2769 & 2345 & 32 \\
& & & & \\
\hline
\end{tabular}


Table 7. Atomic coordinates $\left(\times 10^{4}\right.$ ) and equivalent isotropic displacement parameters $\left(\AA^{2} \times 10^{3}\right)$ for compound 3-b. U(eq) is defined as one third of the trace of the orthogonalized $\mathrm{U}^{\mathrm{ij}}$ tensor.

\begin{tabular}{|c|c|c|c|c|}
\hline & $\mathrm{X}$ & $\mathrm{y}$ & z & $\mathrm{U}(\mathrm{eq})$ \\
\hline $\mathrm{C}(1)$ & $4850(2)$ & $-1446(2)$ & $7595(1)$ & $43(1)$ \\
\hline$C(2)$ & 4979(2) & $-1006(1)$ & $6858(1)$ & $30(1)$ \\
\hline$C(3)$ & $5028(2)$ & $-503(1)$ & $5931(1)$ & $26(1)$ \\
\hline $\mathrm{C}(4)$ & $4127(2)$ & $-886(1)$ & $5034(1)$ & $26(1)$ \\
\hline$C(5)$ & $5912(2)$ & 397(1) & $5873(1)$ & $25(1)$ \\
\hline$C(6)$ & $6999(2)$ & 942(1) & $6743(1)$ & $27(1)$ \\
\hline$C(7)$ & $6365(2)$ & $2045(1)$ & $6713(1)$ & $32(1)$ \\
\hline $\mathrm{C}(8)$ & $6295(2)$ & $2510(1)$ & $5842(1)$ & $34(1)$ \\
\hline C(9) & $6906(2)$ & $1852(1)$ & $5069(1)$ & $30(1)$ \\
\hline$C(10)$ & 8599(2) & $1506(1)$ & $5557(1)$ & 29(1) \\
\hline $\mathrm{C}(11)$ & $9976(2)$ & $1626(1)$ & $5176(1)$ & $40(1)$ \\
\hline$C(12)$ & $11432(2)$ & $1255(2)$ & $5721(2)$ & $48(1)$ \\
\hline$C(13)$ & $11489(2)$ & $783(2)$ & $6629(2)$ & $45(1)$ \\
\hline $\mathrm{C}(14)$ & $10102(2)$ & $662(1)$ & $7017(1)$ & $35(1)$ \\
\hline$C(15)$ & $8653(2)$ & $1018(1)$ & $6470(1)$ & $28(1)$ \\
\hline$C(16)$ & $5910(2)$ & $2450(2)$ & $7644(2)$ & $45(1)$ \\
\hline $\mathrm{C}(17)$ & $5657(3)$ & $3563(2)$ & $5548(2)$ & $55(1)$ \\
\hline $\mathrm{F}(1)$ & $4527(4)$ & $2140(3)$ & $7772(3)$ & $113(2)$ \\
\hline $\mathrm{F}(2)$ & $6025(4)$ & $3451(2)$ & $7746(2)$ & $78(1)$ \\
\hline $\mathrm{F}(3)$ & $6990(3)$ & $2128(2)$ & $8455(1)$ & 69(1) \\
\hline $\mathrm{F}(4)$ & $6577(5)$ & $4296(3)$ & $5968(4)$ & $107(2)$ \\
\hline $\mathrm{F}(5)$ & $5246(5)$ & $3677(2)$ & $4588(2)$ & 86(1) \\
\hline $\mathrm{F}(6)$ & $4264(4)$ & $3744(2)$ & $5884(3)$ & $90(1)$ \\
\hline $\mathrm{F}\left(1^{\prime}\right)$ & $6754(14)$ & $3002(13)$ & $8162(9)$ & $99(5)$ \\
\hline $\mathrm{F}\left(2^{\prime}\right)$ & $4541(14)$ & $3126(7)$ & $7325(8)$ & $86(3)$ \\
\hline $\mathrm{F}\left(3^{\prime}\right)$ & $5229(17)$ & $1760(7)$ & $8051(9)$ & $90(5)$ \\
\hline $\mathrm{F}\left(4^{\prime}\right)$ & $6836(16)$ & $3977(6)$ & $4954(10)$ & $92(3)$ \\
\hline $\mathrm{F}\left(5^{\prime}\right)$ & $4460(30)$ & $3603(11)$ & $4967(18)$ & $164(11)$ \\
\hline$F\left(6^{\prime}\right)$ & $5970(30)$ & $4246(11)$ & $6079(10)$ & $147(12)$ \\
\hline $\mathrm{C}(18 \mathrm{~S})$ & $10670(20)$ & $4605(14)$ & $4839(17)$ & $48(5)$ \\
\hline $\mathrm{Cl}$ & $8716(4)$ & $4431(2)$ & $4320(3)$ & $68(1)$ \\
\hline
\end{tabular}


Table 8. Anisotropic displacement parameters $\left(\AA^{2} \times 10^{3}\right)$ for compound 3-b. The anisotropic displacement factor exponent takes the form: $-2 \pi^{2}\left[\mathrm{~h}^{2} \mathrm{a}^{* 2} \mathrm{U}^{11}+\ldots+2 \mathrm{hk} \mathrm{a}^{*} \mathrm{~b}^{*} \mathrm{U}^{12}\right]$.

\begin{tabular}{lcccccc}
\hline & $\mathrm{U}^{11}$ & $\mathrm{U}^{22}$ & $\mathrm{U}^{33}$ & $\mathrm{U}^{23}$ & $\mathrm{U}^{13}$ & $\mathrm{U}$ \\
& & & & & & \\
\hline $\mathrm{C}(1)$ & $50(1)$ & $46(1)$ & $34(1)$ & $12(1)$ & $8(1)$ & $1(1)$ \\
$\mathrm{C}(2)$ & $31(1)$ & $29(1)$ & $29(1)$ & $1(1)$ & $4(1)$ & $0(1)$ \\
$\mathrm{C}(3)$ & $27(1)$ & $25(1)$ & $26(1)$ & $2(1)$ & $7(1)$ & $2(1)$ \\
$\mathrm{C}(4)$ & $29(1)$ & $22(1)$ & $28(1)$ & $0(1)$ & $7(1)$ & $1(1)$ \\
$\mathrm{C}(5)$ & $26(1)$ & $25(1)$ & $25(1)$ & $-1(1)$ & $6(1)$ & $2(1)$ \\
$\mathrm{C}(6)$ & $31(1)$ & $26(1)$ & $24(1)$ & $-1(1)$ & $6(1)$ & $0(1)$ \\
$\mathrm{C}(7)$ & $32(1)$ & $28(1)$ & $35(1)$ & $-7(1)$ & $7(1)$ & $0(1)$ \\
$\mathrm{C}(8)$ & $36(1)$ & $25(1)$ & $37(1)$ & $-4(1)$ & $2(1)$ & $2(1)$ \\
$\mathrm{C}(9)$ & $36(1)$ & $24(1)$ & $28(1)$ & $2(1)$ & $5(1)$ & $-3(1)$ \\
$\mathrm{C}(10)$ & $33(1)$ & $25(1)$ & $30(1)$ & $-3(1)$ & $7(1)$ & $-3(1)$ \\
$\mathrm{C}(11)$ & $42(1)$ & $41(1)$ & $38(1)$ & $-1(1)$ & $13(1)$ & $-8(1)$ \\
$\mathrm{C}(12)$ & $35(1)$ & $53(1)$ & $59(1)$ & $-3(1)$ & $19(1)$ & $-4(1)$ \\
$\mathrm{C}(13)$ & $31(1)$ & $43(1)$ & $58(1)$ & $-1(1)$ & $5(1)$ & $3(1)$ \\
$\mathrm{C}(14)$ & $36(1)$ & $29(1)$ & $39(1)$ & $1(1)$ & $2(1)$ & $0(1)$ \\
$\mathrm{C}(15)$ & $32(1)$ & $23(1)$ & $30(1)$ & $-2(1)$ & $6(1)$ & $-2(1)$ \\
$\mathrm{C}(16)$ & $50(1)$ & $43(1)$ & $48(1)$ & $-15(1)$ & $18(1)$ & $-1(1)$ \\
$\mathrm{C}(17)$ & $71(1)$ & $31(1)$ & $56(1)$ & $-3(1)$ & $-9(1)$ & $11(1)$ \\
$\mathrm{F}(1)$ & $67(2)$ & $187(4)$ & $102(2)$ & $-94(3)$ & $55(2)$ & $-62(2)$ \\
$\mathrm{F}(2)$ & $131(3)$ & $43(1)$ & $65(1)$ & $-18(1)$ & $29(1)$ & $16(1)$ \\
$\mathrm{F}(3)$ & $92(2)$ & $80(1)$ & $33(1)$ & $-13(1)$ & $7(1)$ & $26(1)$ \\
$\mathrm{F}(4)$ & $117(2)$ & $26(1)$ & $146(4)$ & $2(2)$ & $-49(2)$ & $-8(1)$ \\
$\mathrm{F}(5)$ & $153(4)$ & $49(1)$ & $49(1)$ & $12(1)$ & $1(2)$ & $41(2)$ \\
$\mathrm{F}(6)$ & $87(2)$ & $73(2)$ & $109(2)$ & $5(1)$ & $15(2)$ & $51(1)$ \\
$\mathrm{F}\left(1^{\prime}\right)$ & $73(6)$ & $147(11)$ & $93(8)$ & $-92(8)$ & $54(6)$ & $-75(7)$ \\
$\mathrm{F}\left(2^{\prime}\right)$ & $102(7)$ & $66(5)$ & $113(7)$ & $10(5)$ & $78(6)$ & $40(5)$ \\
$\mathrm{F}\left(3^{\prime}\right)$ & $160(13)$ & $46(4)$ & $102(8)$ & $3(4)$ & $119(10)$ & $-1(6)$ \\
$\mathrm{F}\left(4^{\prime}\right)$ & $133(9)$ & $32(4)$ & $128(8)$ & $33(4)$ & $67(7)$ & $9(4)$ \\
$\mathrm{F}\left(5^{\prime}\right)$ & $165(16)$ & $76(8)$ & $179(19)$ & $42(12)$ & $-138(15)$ & $-14(10)$ \\
$\mathrm{F}\left(6^{\prime}\right)$ & $340(30)$ & $47(8)$ & $45(5)$ & $-27(5)$ & $24(11)$ & $80(13)$ \\
$\mathrm{C}(18 \mathrm{~S})$ & $46(10)$ & $33(9)$ & $73(13)$ & $-20(9)$ & $35(10)$ & $-6(8)$ \\
$\mathrm{Cl}$ & $74(2)$ & $60(2)$ & $70(2)$ & $-7(1)$ & $14(2)$ & $-8(1)$ \\
& & & & & & \\
\hline & & & & & &
\end{tabular}


Table 9. Hydrogen coordinates ( x 10 $0^{4}$ ) and isotropic displacement parameters $\left(\AA^{2} \times 10^{3}\right)$ for compound 3-b.

\begin{tabular}{lrrrr}
\hline & $x$ & $y$ & $z$ & U(eq) \\
\hline $\mathrm{H}(1)$ & 4746 & -1799 & 8185 & 52 \\
$\mathrm{H}(6)$ & 7028 & 597 & 7398 & 33 \\
$\mathrm{H}(9)$ & 6864 & 2213 & 4421 & 35 \\
$\mathrm{H}(11)$ & 9933 & 1955 & 4553 & 47 \\
$\mathrm{H}(12)$ & 12388 & 1328 & 5467 & 57 \\
$\mathrm{H}(13)$ & 12489 & 537 & 6995 & 54 \\
$\mathrm{H}(14)$ & 10149 & 341 & 7644 & 42 \\
$\mathrm{H}(18 \mathrm{~A})$ & 11248 & 4701 & 4286 & 57 \\
$\mathrm{H}(18 B)$ & 11062 & 3955 & 5171 & 57 \\
\hline
\end{tabular}

